\title{
RUPTURA DEL SENO MARGINAL DE LA PLACENTA
}

\author{
Dr. Luis Alba Palacios \\ Dr. Bernardo Handzer $G$. \\ Dra. Elsa Valderrama
}

La ruptura del seno marginal de la placenta ocurrida en el curso del embarazo, es un accidente que a pesar de ser relativamente frecuente, suele quedar ignorado; el hecho de que la hemorragia que ocasiona no adquiera caracteres dramáticos, ni imponga, por lo tanto, procedimientos terapéuticos extremos, ha contribuído a que la entidad pase desapercibida 0 se confunda con otros problemas obstétricos o ginecológicos; es probable que en muchos hospitales de maternidad la ruptura del seno marginal de la placenta no haya figurado nunca en los ficheros estadísticos, pero, en cambio, es indudable que un buen número de casos rotulados como placenta previa o abruptio placentae, sean evidentemente rupturas del seno marginal.

En vista de que en nuestro medio no existe ninguna publicación acerca de esta entidad, emprendimos su estudio en el Instituto Materno Infantil "Concepción Villaveces de Acosta", de Bogotá, con el objeto de comprobar los hallazgos de autores extranjeros $y$, especialmente, con la intención de despertar interés por el diagnóstico y manejo de dicha complicación del embarazo.

\section{Historia}

Según Ferguson la descripción más antigua que se conoce de la ruptura del seno marginal de la placenta, fué hecha por Jacquemier, en Francia, en 1839. Este autor pensó que era ocurrencia frecuente tanto en los embarazos normales como en aquellos complicados con desprendimiento prematuro de la placenta o con inserción previa. En Edimburgo, Duncan y Simpson en 1870, inculparon a la ruptura del seno marginal como una de las causas de hemorragia observada al iniciarse el trabajo de parto. En 1893, Budin, en París publicó algunos dibujos que describían exactamente el problema. Posteriormente el abruptio y la placenta previa mantuvieron en el olvido al seno marginal como elemento potencialmente productor de hemorragia en la gestación tardía. En 1935, Spanner despertó nuevamente la atención al observar el seno marginal en placentas todavía adheridas al útero, utilizando el método de la corrosión. La demostración de la comunicación del seno marginal con los senos venosos maternos, fue debida a B. Harris en 1952, también estudiando placentas in situ. En 1958 Schneider hizo su descripción anatómica detenida y W. 
Coullier se ocupó de los aspectos clínicos. J. De Rezende, R. Rocco y Barcellos, en 1958 destacaron la importancia de la histopatología para el diagnóstico. Por último, los trabajos de Ferguson (1951, 1956, 1959) y los de Fish y colaboradores, hicieron de la ruptura del seno marginal una entidad clínica e histopatológica definida.

\section{DESARROLLO DE LA CIRCULACION PLACENTARIA}

\section{Embriología}

Para comprender la embriología de la circulación placentaria, tenemos que estudiarla a partir de los primeros días de desarrollo del huevo fertilizado. Se sabe que la primera semana, este huevo fertilizado sufre una serie de divisiones celulares hasta su organización en blastómeras y la formación de una estructura quística, el blastocisto, el que está constituído por una masa interna de cé-
Iulas germinales y una cápsula, el trofoblasto. Al final del 60 día esta estructura se adhiere al epitelio del útero y empieza a hundirse dentro de la mucosa. La pared trofoblástica constituída inicialmente por células citotrofoblásticas, prolifera, las células pierden sus límites y se convierten en sincicio, el que se coloca periféricamente $y$ se pone en contacto con el tejido conectivo del endometrio.

Aproximadamente al 9: día, el trofoblasto o futuro corión inicia su desarrollo especializado para favorecer el intercambio feto-materno. En las células sinciciales aparecen vacuolas, las que se van agrandando y uniéndose con las vecinas formando espacios irregulares o lagunas las que se comunican entre sí en forma laberíntica y comunicándose rápidamente con los vasos sanguíneos del útero. Estas lagunas son los que darán origen a los futuros espacios intervellosos. (Figs. Nos. 1 y 2 ).

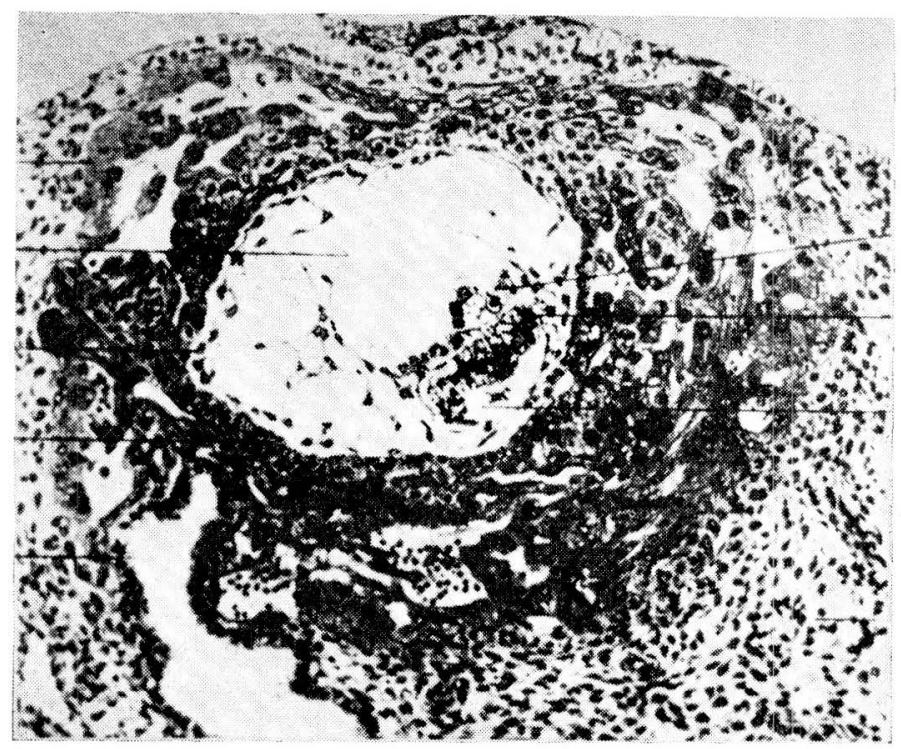

FIGURA $N^{0} 1$ - Embrión de nueve días de desarrollo. Iniciación de la formación de vacuolas a partir de las células sinciciales. (Arey). 


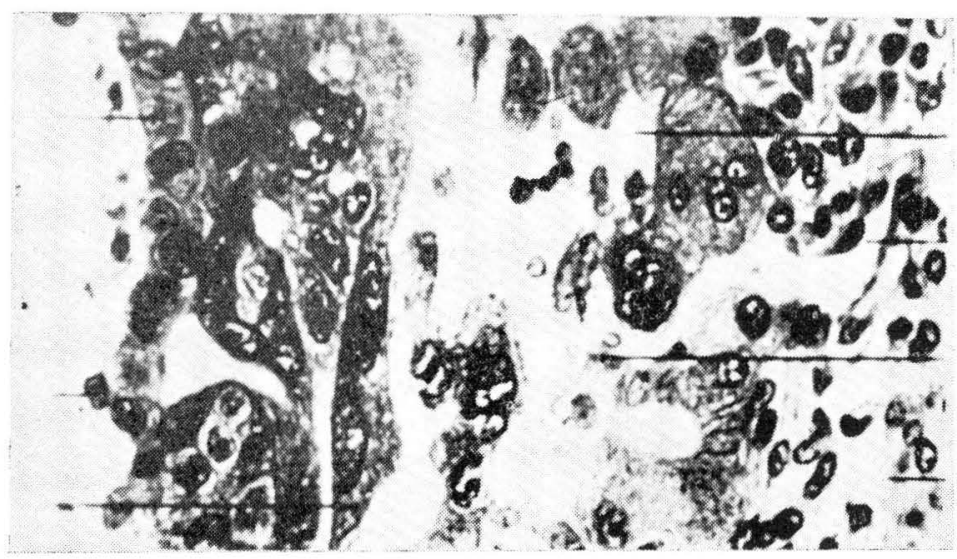

FIGURA N $N^{0} 2$ - Trofoblasto de once días de desarrollo embrionario, lagunas sanguíneas completamente formadas y anastomosadas. (Arey).

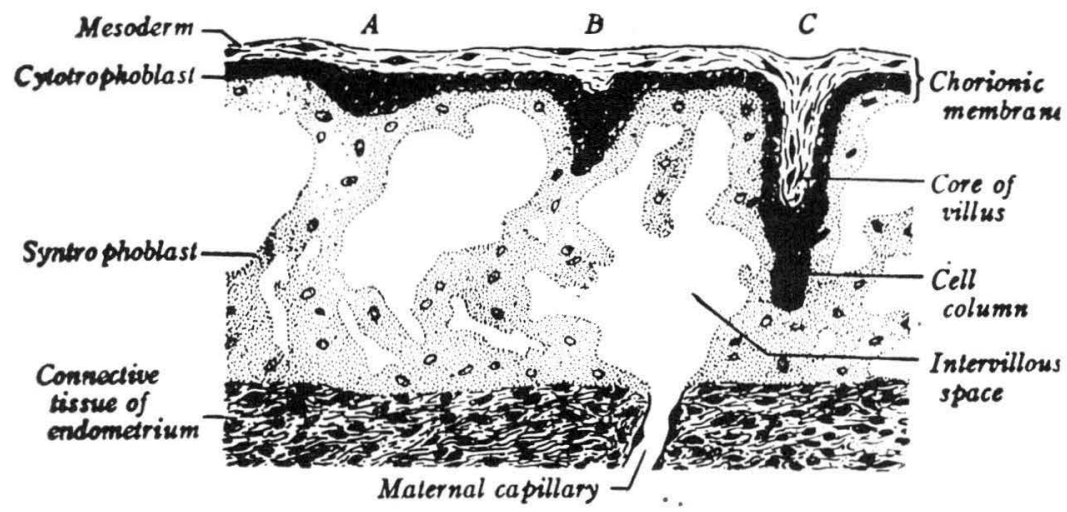

FIGURA No 3 - Embarazo de trece días de desarrollo, muestra la proliferación de células citotrofoblásticas y la iniciación de los tallos conjuntivos. (Arey).

Al 13 día de desarrollo, el primitivo mesodermo extraembrionario, se consolida en una sola capa definida por debajo del trofoblasto, para dar origen al corion. Por su cara externa, esta membrana está cubierta por una sola hilera de células citotrofoblásticas, las que empiezan a proliferar en forma localizada a partir del 16: día del desarrollo embrionario. Al mismo tiempo se desarrollan pequeños tallos de tejido mesodérmico del corion, los que se introducen en el centro de estas proliferaciones celulares, siendo al principio muy pequeños, van creciendo progresivamente a medida que el citotrofoblasto prolifera, produciéndose ramificaciones en diferentes direcciones. Los vasos sanguíneos fetales, que corren por la placa coriónica, se continúan en estas ramificaciones. Al final de la $3^{a}$ semana las vellosidades están completamente constituídas y las recubre una doble hilera de células trofoblásticas, una interna citotrofo- 


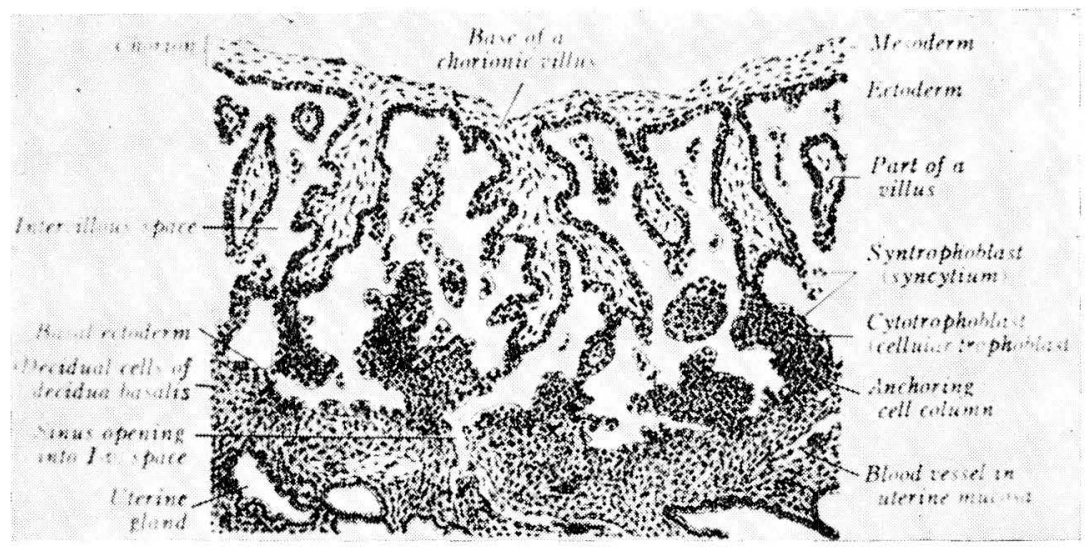

FIGURA No 4 - Embarazo de tres semanas, desarrollo completo de las vellosidades coriales. (Arey).

blástica y una externa, sinciciotrofoblástica, estas células son las que van a formar el revestimiento de los espacios intervellosos por donde circulará la sangre materna y permitirán el intercambio de sustancias nutritivas de la madre al feto.

\section{CIRCULACION PLACENTARIA}

\section{Reseña histórica}

El estudio de la circulación placentaria se remonta a tiempos muy lejanos y presenta períodos de extraordinaria importancia por la grandeza de los trabajos. Es a estos períodos que nos referimos en esta breve reseña histórica.

La primera comunicación, al parecer fue hecha por Galeno (A.D. 130200 q.v.) quien al describir el útero embarazado decía: "Los vasos internos del útero abren las bocas y se unen con los vasos fetales en la cubierta externa del embrión, (corion), y lo hacen de tal manera, que se unen vasos de la misma clase, es decir, arterias con arterias y venas con venas, formándose igual número de vasos a los existentes en el útero".
En esta forma se constituía una sola circulación y la sangre materna circulaba a través del feto como si fuera parte de su propio cuerpo.

El siglo XVIII, se caracterizó por los estudios de grandes investigadores, en 1741 William Noortwck, inyecta por primera vez, un útero embarazado. Al disecar el órgano encuentra el líquido inyectado bajo la superficie fetal de la placenta y al examinarla por la cara materna encontró múltiples vasos inyectados, los que se prolongaban hasta la decidua y el útero. El creyó, como hasta ahora se había dicho, que estos eran vasos fetales que iban hasta el útero para conseguir la nutrición del embrión.

En 1754, Colin Mackenzie en colaboración con John Hunter, con el ánimo de repetir las experiencias de Noortwyck, practican la autopsia a 13 pacientes e inyectan las placentas, disecan cuidadosamente las ramificaciones vasculares y comprueban que los vasos maternos son los que Ilegan al tejido placentario. Se puede decir que este es el primer trabajo 
que define la parte materna de la circulación placentaria, estos investigadores son los primeros en describir en sus trabajos la característica espirilada de las arterias uterinas. No inyectaron los vasos fetales, pero hacen una descripción similar a la conocida hoy.

A estos investigadores se les considera actualmente como a los verdaderos iniciadores del estudio de la circulación placentaria, ya que todos sus hallazgos han sido confirmados y la base de los estudios modernos, tienen sus principios en los trabajos realizados por ellos.

El estudio de las vellosidades coriales y de la circulación fetal se inicia en 1830 con el descubrimiento del microscopio, pero es Ernst Heinrich Weber de Leipzig, quien hace una descripción detallada y además describe la cubierta trofoblástica de las vellosidades.

En 1873 William Turner, demuestra que la placenta de los monos tienen igual estructura que la pla- centa humana, abriéndose así un amplio campo para la investigación.

El último gran período se inicia en 1893 con los trabajos realizados por Wilhem Waldeyer, anatomista y Ernst Bumm, ginecólogo. El trabajo lo realizan examinando directamente y haciendo estudio histológico de las placentas. Estos autores dicen que cada cotiledón materno es una mitad circulatoria independiente, las arterias maternas corren a través de los septos, abriéndose al espacio intervelloso por el borde o por el ápice de estos septos, luego la sangre es drenada por las venas localizadas en la serotina o decidua basal, las que comunican con los senos venosos uterinos. Ellos demuestran que solo hacia el espacio subcoriónico se comunican entre sí estas unidades circulatorias y que a nivel del cotiledón marginal, se comunican con el así llamado "Seno Marginal" al cual solo se le puede atribuír papel secundario para el drenaje de la sangre de los cotiledones centrales. Este trabajo es muy importante debido a que actualmente es aceptado por muchos investigadores. (Fig. № 5 ).

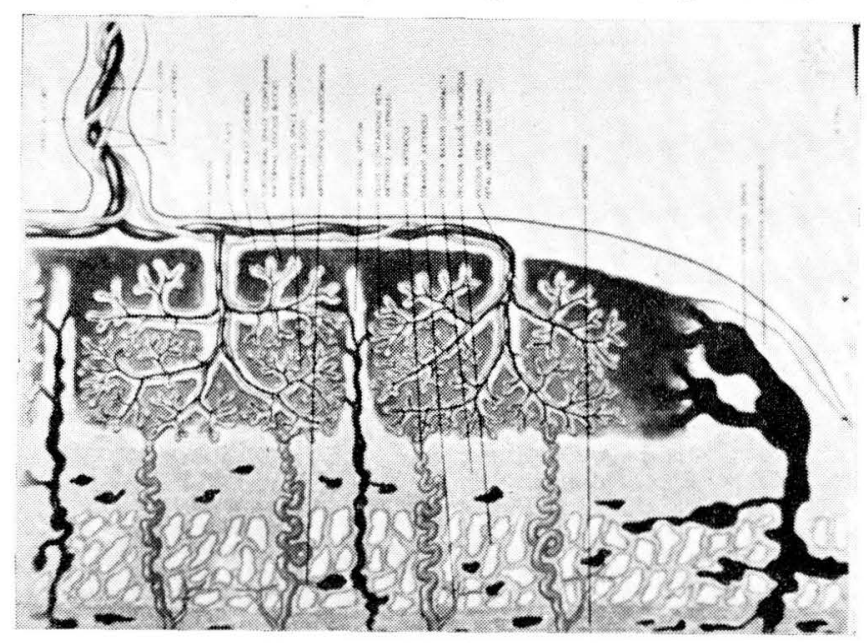

FIGURA No 5 Diagrama de la circulación placentaria. 
En 1935 R. Spanner publica sus propias experiencias, negando los conceptos de Bumm. El trabajo de este autor se basa en la inyección de sustancias plásticas, tanto en la sangre materna como en la fetal, en placentas in-situ y en placentas expulsadas en diferentes edades del desarrollo del embarazo.

El observa la entrada de la sangre arterial por la placa basal, la cual es guiada al espacio subcorial por septos no comunicantes, la sangre drena lateralmente al margen placentario en donde existe el solo medio de comunicación con las venas subplacentarias maternas, esta estructura recibe el nombre de Seno Marginal.

Esta teoría fue ampliamente aceptada por anatomistas, embriólogos y obstetras, ya que con este mecanismo de la circulación placentaria se explicaba en forma lógica la hemorragia patológica durante el embarazo.

Son muchos los estudios que se han hecho en los últimos años, para confirmar las teorías anteriores y dejar una como clásica, que sea aceptada mundialmente, pero no se ha llegado a un concepto definitivo. La Dra. Elizabeth Ramsey, siendo una de las más interesadas en estos problemas y no aceptando la teoría de Spanner, dice refiriéndose a la función del Seno Marginal: "Puede representar un área en la cual la sangre llega a estar congestionada bajo circunstancias desusuales y aún patológicas, así podría ascribírsele a esta estructura el papel de una válvula de seguridad en casos de emergencia, más que una importancia primaria en el drenaje venoso". (Fig. No 6).

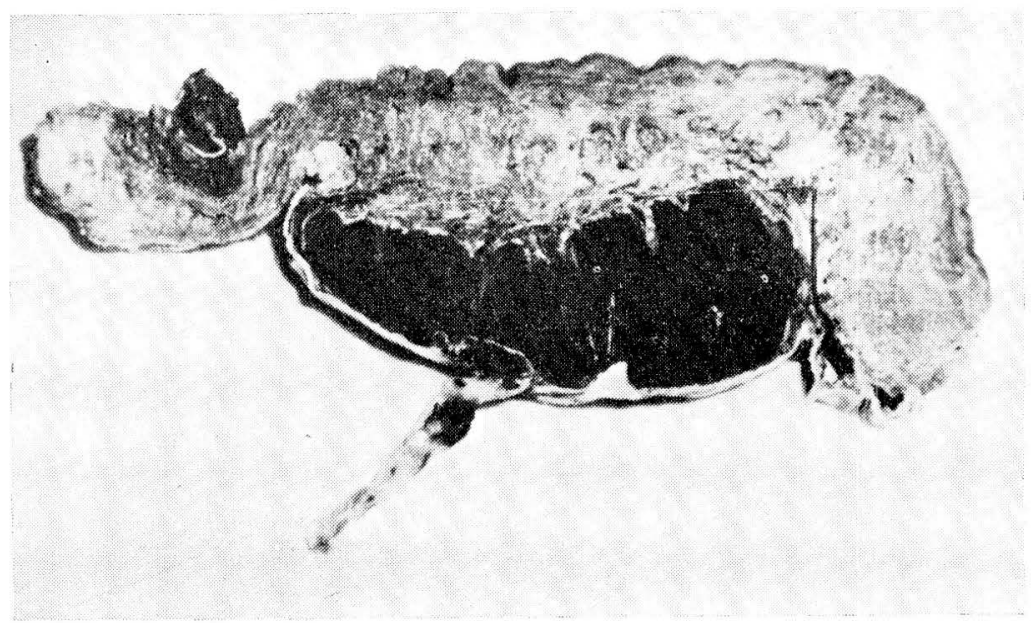

FIGURA No 6 - Placenta In Situ.

Observar el seno marginal de la placenta.

Según el trabajo de Crottogine, Zubiaurre, (Mar del Plata), y CaldeyroBarcia presentado en el IV Congreso de Obstetricia y Ginecología, se demuestra que el drenaje venoso placentario puede hacerse por vía basal o por el seno marginal, dependiendo de la presión existente en el espacio intervelloso. Ellos demostraron al inyectar un medio de contraste (Hypaque) en este espacio y controlando continuamente por televisión, que 
cuando se inyectaba lentamente (1 cc. por minuto), la sangre drenaba por las venas del mismo cotiledón, pero si la inyección era rápida (5 cc. por minuto), el contraste pasaba a los cotiledones vecinos y a través del lago subcorial al seno marginal. Los autores comentan que las vías de drenaje venoso de la placenta no son exclusivas como las describieron Bumm y Spanner sino que ambos mecanismos pueden coexistir asegurando la integridad de la circulación placentaria.

Es en base de estos conceptos que varios autores han descrito el desgarro del Seno Marginal como una entidad clínica, producida por múltiples factores que alteran ya, la presión de la circulación en los espacios intervellosos o cambios a nivel uterino que producen trauma sobre esta delicada estructura marginal como son las contracciones uterinas, la formación del segmento, borramiento y dilatación del cuello o aún el mismo feto puede producir presión y estiramiento de las membranas, las que van a obrar sobre el margen placentario produciendo desprendimiento de éste y desgarro del seno. Este cuadro se asocia generalmente a las placentas con inserción baja lesionándose en su borde inferior.

\section{Material y Métodos}

Entre el 1\% de Mayo y el 31 de Diciembre de 1965, se estudiaron cuidadosamente 118 casos con hemorragia genital aparecida durante el tercer trimestre del embarazo. De ellas $41(34,7 \%)$ correspondieron a ruptura del seno marginal de la placenta, y son el motivo de la presente publicación.

Para efecto de clasificar la causa y el tipo de hemorragia, a cada paciente se llevó un registro especial en donde se consignaron los siguientes datos:

a) Momento de iniciación de la hemorragia genital (anteparto, intraparto, si en el período de dilatación o en el período expulsivo);

b) Cantidad de la hemorragia, calculada como abundante (500 cc. o más), moderada (de 200 a 400 cc.) y escasa (menos de 200 cc.);

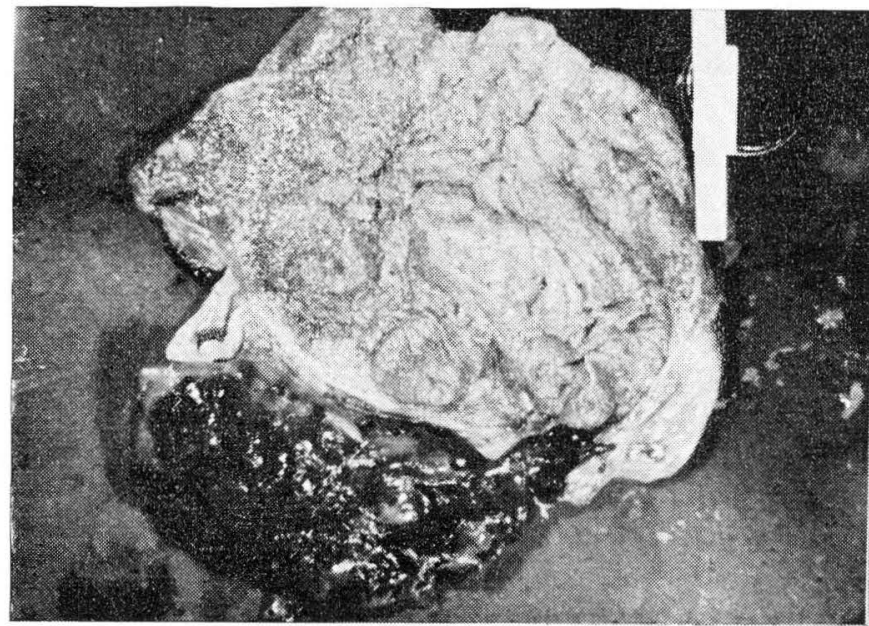

FIGURA $\mathbf{N}^{0} 7$ 
c) Alteraciones de la dinámica uterina (hipertonía o polisistolia);

d) Dolor abdominal;

e) Estado del feto al aparecer la hemorragia $o$ en el momento del ingreso;

f) Signología de toxemia.

El criterio clínico para considerar que la hemorragia había sido causada por la ruptura del seno marginal de la placenta, se basó en la presencia de hemorragia genital indolora y en la eliminación de la placenta pre- via con el tacto vaginal. La comprobación se hizo cuando el examen en fresco de las placentas mostró coágulo organizado, adherido al borde de la placenta, sobre una solución de continuidad del seno marginal y que se prolongaba dentro de éste. (Fig. № 7).

La identificación de una ruptura de la pared del seno marginal, no fue por sí sola elemento para el diagnóstico; es indispensable el hallazgo de un trombo superficial que se continúa dentro de la cavidad del seno. (Fig. No 8).

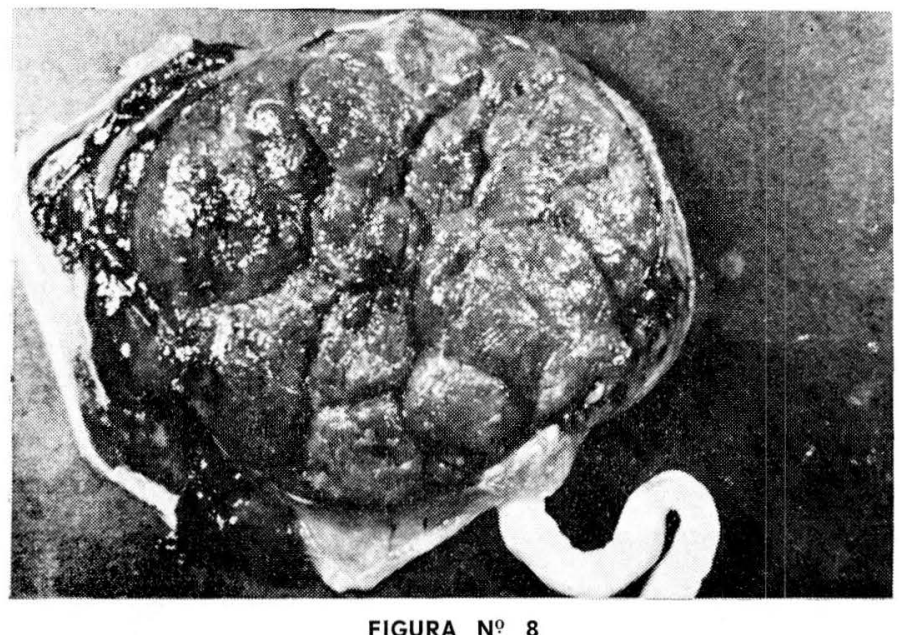

\section{Resultados}

De los 41 casos estudiados, en 19 la hemorragia apareció antes de la iniciación del trabajo de parto, $(46,3 \%)$, en 21 durante el período de dilatación y en 1 en la fase expulsiva $(53,7 \%)$.

La hemorragia fue manifestación única de la ruptura del seno marginal en 31 casos, es decir en las tres cuartas partes de los casos. El dolor la acompañó en 6 y la hipertonía en 4.
CUADRO N $N 1$

SIGNOS Y SINTOMAS ASOCIADOS

\begin{tabular}{lrr}
\hline & Casos & Porcentaje \\
\hline Hemorragia & 31 & $75,7 \%$ \\
Hemorragia - dolor & 6 & $14,6 \%$ \\
Hemorragia - hipertonía & 4 & $9,7 \%$ \\
\hline
\end{tabular}

La cantidad de sangre expulsada se catalogó como escasa en 18 oportunidades, moderada en 17 y abundante únicamente en 6 casos. 
CUADRO N: 2

CANTIDAD DE LA HEMORRAGIA

\begin{tabular}{lllll}
\hline & Casos & Porcentaje \\
\hline Abundante (400 cc. 0 más) & 6 & $14,6 \%$ \\
Moderada ( 200 a 400 cc.) & 17 & $41,5 \%$ \\
Escasa (200 cc. 0 menos) & 18 & $43,9 \%$ \\
\hline
\end{tabular}

Al ingresar las pacientes al hospital el estado del feto era bueno en 32 casos, mientras que 2 presentaban signos de sufrimiento y siete ingresaron muertos.

CUADRO N: 3

ESTADO FETAL AL INGRESO

\begin{tabular}{lcc}
\hline & Casos & Porcentaje \\
\hline Bueno & 32 & $78,0 \%$ \\
$\begin{array}{l}\text { Regular } \\
\text { (signos de sufrimiento) }\end{array}$ & 2 & $5,0 \%$ \\
Muerto & 7 & $17,0 \%$ \\
\hline
\end{tabular}

Encontramos que fue más frecuente este accidente en el grupo de pacientes entre los 20 a los 30 años, seguido del de 31 a 40 años.

\section{CUADRO NN 4}

EDAD DE LAS PACIENTES

\begin{tabular}{lrlr}
\hline & Casos & Porcentaje \\
\hline Menos de 19 años & 1 & $2,5 \%$ \\
De 20 a 30 años & 25 & $61,0 \%$ \\
De 31 a 40 años & 15 & $36,5 \%$ \\
\hline
\end{tabular}

Según la edad del embarazo, el grupo que con mayor frecuencia presentó esta complicación fué el comprendido entre 37 y 40 semanas ( 26 casos), seguido del de 28 a 36 semanas ( 13 casos). En los grupos de 20 a 27 semanas, así como el de 41 o más, solo figura un caso en cada uno.
CUADRO № 5

EDAD DEL EMBARAZO

\begin{tabular}{|c|c|c|}
\hline & Casos & Porcentaje \\
\hline 28 a 27 semanas & 1 & $2,4 \%$ \\
\hline 28 a 36 & 13 & $31,7 \%$ \\
\hline 37 a 40 & 26 & $63,5 \%$ \\
\hline 41 ○ más semanas & 1 & $2,4 \%$ \\
\hline
\end{tabular}

En el cuadro correspondiente a la paridad ( $N$ : 6), observamos que el grupo de 5 partos o más, figuran 27 casos; en el de 3 partos, 6 casos y en el de las primíparas 5 casos.

\section{CUADRO N: 6}

\begin{tabular}{lrr}
\multicolumn{3}{c}{ PARIDAD } \\
\hline & Casos & Porcentaje \\
\hline Uno & 5 & $12,1 \%$ \\
Dos & 2 & $4,8 \%$ \\
Tres & 6 & $14,6 \%$ \\
Cuatro & 1 & $2,5 \%$ \\
Cinco o más & 27 & $66,0 \%$ \\
\hline
\end{tabular}

El estado del feto al nacimiento, fue bueno en 24 casos, clasificados con Apgar de 7 a 10/10; regular, con Apgar de 4 a 6/10, 6 casos. Dos nacieron en malas condiciones, Apgar 1 a 3/10 y 9 nacieron muertos.

\section{CUADRO $\mathrm{N}: 7$} ESTADO DEL FETO AL NACER

\begin{tabular}{lrrr}
\hline & Casos & Porcentaje \\
\hline Bueno (Apgar 7 a $10 / 10)$ & 24 & $58,5 \%$ \\
Regular (Apgar 4 a $6 / 10)$ & 6 & $14,7 \%$ \\
Malo (Apgar de 1 a $3 / 10)$ & 2 & $4,8 \%$ \\
Muerto & 9 & $22,0 \%$ \\
\hline
\end{tabular}

Los fetos tuvieron un peso de 1.000 a 2.499 gramos en 17 casos $(41,5 \%)$, de 2.500 gramos o más, en 24 casos $(58,5 \%)$.

Los partos fueron espontáneos en 38 casos $(92,6 \%)$ y se requirió la intervención cesárea en los 3 restantes $(7,4 \%)$. 


\section{Comentarios}

La ruptura del seno marginal, como hemos podido apreciar, determina hemorragia genital externa como signo único en las tres cuartas partes de los casos $(75,7 \%)$, lo cual es capital para el diagnóstico diferencial con otras entidades hemorrágicas del último trimestre del embarazo. Observamos su asociación con dolor en una cuarta parte de los casos, el cual fue siempre de carácter moderado o leve. Solamente 4 casos presentaron hemorragia e hipertonía uterina, también de moderada intensidad. En ninguno de los casos observamos la tríada de hemorragia, dolor e hipertonía, tan frecuente en el abruptio. Ferguson y Haton en su interesante trabajo sobre abruptio placentae $y$ ruptura de seno marginal, hacen anotaciones semejantes acerca de la sintomatología.

Fish y colaboradores, y F. Ripley encontraron que la ruptura del seno marginal se produce al hacer su aparición las contracciones uterinas dolorosas y la mayoría de las veces en el período de dilatación, lo cual está de acuerdo con lo observado por nosotros, pues la hemorragia apareció en el $53,7 \%$ de los casos una vez que el trabajo de parto se había iniciado.

Generalmente la sangre es de aspecto rutilante, en cantidad moderada o escasa, no sobrepasando los 200 cc., y, por lo tanto, no poniendo en peligro la vida fetal o materna. Ninguna de nuestras pacientes mostró signos de anemia aguda. Teniendo en cuenta que la sangre contenida en el seno marginal es de origen materno, lógicamente la amenaza para la vida fetal es muy remota. En los casos estudiados el estado fetal al ingreso de la paciente fué bueno en el $78 \%$ de los casos; un $5 \%$ pre- sentó signos de sufrimiento fetal, pero las madres estaban en buenas condiciones generales. Siete fetos $(17 \%)$ fueron hallados muertos al practicar la historia clínica de ingreso de la madre; en ninguno de estos casos la hemorragia fué de intensidad suficiente como para explicar la muerte fetal. Hay que tener en cuenta que de las 41 pacientes que estudiamos 11 tenían alguna forma de toxemia.

Relacionando la R. S. M. con la edad de las pacientes, encontramos, como era de esperar, que es más frecuente en el grupo de 20 a 30 años $(61 \%)$, que corresponde igualmente al grupo de mayor actividad reproductiva. En cuanto a la paridad, Ilama poderosamente la atención el predominio de este accidente en el grupo de pacientes multíparas con cinco - más partos (66\%). Estos datos fueron observados igualmente en grupos estudiados de placentas previas y abruptio placentae, cuyo análisis será motivo de un trabajo posterior.

Un hecho interesante es la asociación muy frecuente de ruptura del seno marginal y prematurez. Ferguson y Haton hallaron esta asociación en el $46 \%$ de sus casos; nosotros, basados en el peso del recién nacido, la encontramos en el $41,5 \%$. Puede presumirse entonces que el desgarro del seno marginal está íntimamente ligado con el parto prematuro y viene a constituir por esta razón una real amenaza para el porvenir fetal. Esta relación, entre nosotros, no ha sido tenida en cuenta para explicar las causas del parto prematuro. En cambio, para Fish y colaboradores constituye la segunda causa después de la ruptura prematura de las membranas.

En cuanto a la vida fetal el pronóstico es bueno, habiéndose obteni- 
do un $73,2 \%$ de fetos en condiciones generales aceptables. Nacieron $9 \mathrm{fe}$ tos muertos $(22 \%)$, de los cuales 7 lo estaban al momento del ingreso, como dijimos anteriormente. Los 2 restantes murieron durante el trabajo de parto y se atribuyó su muerte más a prematurez que a la ruptura del seno marginal. Depurando pues la mortalidad fetal, no encontramos ningún caso de muerie atribuíble exclusivamente a la ruptura del seno marginal de la placenta.

No encontramos mortalidad materna atribuíble a ruptura de seno marginal, coincidiendo en esto con los diferentes autores que han estudiado este síndrome. En los casos estudiados hubo solamente una muerte materna en una paciente nefrótica grave, cuya lesión renal fue la causa de la muerte.

La toxemia se presentó en concomitancia con la ruptura del seño marginal en el 26,8\%. Nelson, Brever, citados por Ferguson, y Ferguson, Haton encuentran una incidencia del $14,4 \%$ y del $17 \%$ respectivamente; esta diferencia nos la explicamos por el alto índice de toxemia en nuestro medio.

El parto fue espontáneo en el $92,6 \%$, y solo requirió cesárea por la severidad de la hemorragia en tres casos $(7,4 \%)$. En ninguna oportunidad, observamos complicaciones intraparto o postparto del tipo del shock 0 de la hipofibrinogenemia. Solamente en 9 casos hubo necesidad de aplicar transfusión sanguínea. Se apeló al recurso de la amniotomía en 15 casos. La inducción con ocitócicos se utilizó en un solo caso y el refuerzo en 8. Los sedantes se emplearon en 11 casos y corresponden a aquellos catalogados como "toxemias".

\section{Diagnóstico}

Para una adecuada identificación de este síndrome, insistimos en la conveniencia de que el obstetra piense en ella cuando se enfrente a una hemorragia genital aparecida en el último trimestre del embarazo. Entre éstas las más importantes son el abruptio placentae y la placenta previa. Su diferenciación con el abruptio debe hacerse teniendo en cuenta el estado general de la paciente y la vitalidad fetal, generalmente no comprometidos por la ruptura del seno marginal y seriamente afectados por el abruptio. La tríada sintomatológica del abruptio -hemorragia, dolor, e hipertonía-, no está nunca presente en el caso de la ruptura del seno marginal; solamente en algunos casos aislados puede observarse, además de la hemorragia, bien sea dolor o hipertonía y siempre de moderada intensidad. El abruptio se asocia de manera casi constante con la toxemia gravídica, mientras que la ruptura del seno marginal lo hace con una frecuencia muy inferior.

Respecto de la placenta previa su diferenciación básicamente se hace por la cantidad de la hemorragia, generalmente abundante en ésta, y moderada o escasa en ruptura de seno marginal. De ahí que la anemia aguda sea frecuente en la placenta previa, poniendo en peligro la vida de la paciente y la del feto, mientras que de existir en la ruptura del seno marginal casi nunca constituye una seria amenaza. Su identificación puede hacerse al practicar el examen vaginal, palpando los cotiledones o el borde placentario a través del cuello, mientras en la ruptura del seno marginal esto no es posible.

La comprobación de la ruptura del seno marginal se hace estudiando la placenta después del alumbramiento; 
ha de observarse un coágulo sobre un desgarro del seno marginal y que se continúa dentro de éste.

\section{Manejo}

En los casos en los cuales la hemorragia es indolora, y el diagnóstico presuntivo es de ruptura del seno marginal, debe seguirse una conducta expectante, excepto cuando la hemorragia comprometa la vida fetal o materna.

La amniotomía es recomendable para tratar de controlar la hemorragia; este es un recurso que debe utilizarse, de ser posible, en cualquier entidad hemorrágica del último trimestre del embarazo.

Si la hemorragia ocurre en una etapa temprana del último trimestre, y no es posible el diagnóstico de placenta previa o de ruptura de seno marginal, por encontrarse el cuello cerrado, la conducta debe ser así mismo expectante, procediendo únicamente a reponer la pérdida sanguínea. Se ha observado que con esta conducta no hay aumento de la mortalidad fetal o materna; al contrario, la prolongación del embarazo permitirá una mayor madurez fetal. La inducción o el refuerzo del trabajo de parto, no tienen cabida como tratamiento específico de la entidad; deberán aplicarse si existe otra indicación para hacerlo. Durante el parto, bien sea por vía vaginal o abdominal, debe manejarse con extremo cuidado la placenta para evitar la ruptura traumática del seno, la disgregación o dislocación de los coágulos y el escape de sangre del seno marginal; hay que tener presente que para poder confirmar o descartar el diagnóstico, es necesario estudiarla en fres$\mathrm{co}$, inmediatamente después del alumbramiento. No es aconsejable aplazar su estudio ni su conservación en ne- vera o coagulador, por cuanto la putrefacción se inicia rápidamente.

\section{Conclusiones}

De nuestras propias observaciones podemos concluír que la ruptura del seno marginal es una entidad obstétrica definida y que, por lo tanto, debe tenerse en cuenta en el diagnóstico diferencial de las hemorragias del último trimestre de la gestación. En nuestro estudio fue causa de hemorragia en el $34,7 \%$ de los casos.

El signo primordial para el diagnóstico es la hemorragia genital moderada o escasa, que raramente compromete el estado materno o fetal y que aparece con más frecuencia durante el curso del trabajo de parto.

Es una entidad que suele complicar el curso de la gestación en las grandes multíparas y es causa muy frecuente de partos prematuros.

La conducta debe ser siempre expectante en el período anteparto; en el curso del parto la amniotomía es un recurso que debe utilizarse. Aunque el parto se sucede casi siempre en forma espontánea, es posible tener que recurrir a la operación cesárea para controlar la severidad de la hemorragia.

Finalmente, debe establecerse como norma la revisión cuidadosa de la placenta en todos aquellos casos de hemorragia genital en el curso del último trimestre del embarazo. Solo así será posible clasificar con veracidad las causas que la provocan.

\section{Resumen}

Se estudiaron 41 casos de ruptura de seno marginal de la placenta, en el I.M.I. "Concepción Villaveces de Acosta", de Bogotá. Se comprueban los hallazgos de otros autores y se hace nalgunas observaciones sobre la 
gran importancia de este síndrome y su forzada inclusión dentro del diagnóstico diferencial de las hemorragias del último trimestre del embarazo. Se analiza su incidencia, la importancia de los síntomas y su pronóstico materno fetal. Se exponen algunas consideraciones básicas en el diagnóstico y manejo.

\section{BIBLIOGRAFIA}

1 ARCY L. B. Revelopment Anatomy. W. B. Saunders \& Co. 1962.

2 BUMM ERNST. The development of the circulation of the maternal blood in the human placenta. A.M.J. Obst. Gyn. 87.829. 1963.

3 BOURNE G. The Human Amnion and Chorion. Year Book medical publishers, Inc. 1962.

4 CORNER GEORGE W. Exploring the placental maze. Am. J. Obst. \& Gyn. 86.408. 1963.

5 CROTTOGINI J. J., ZUBIAURRE L., CALDEYRO-BARCIA R. Circulación útero placentaria en el embarazo humano. Trabajo presentado al 40 Congreso Mundial de la Federación de Ginecología y Obstetricia. Mar del Plata. Sep. 1964.

6 DE REZENDE J., NAHOUM J. C., BARCELLOS J. M., BELFORT P. La Placenta y los nuevos caminos de la Obstetricia. Rev. Sandorama. Julio 1965.

7 FERGUSON J. H., MILLER, H. K. Toxemia of pregnancy at Charity Hospital in New Orleans. Surg., Gyn. \& Obst. 101: 257-268. 1955.
8 FERGUSON J. H. Late Pregnancy bleeding and rupture of the marginal sinus, New England Journal of Medicine. Vol. 254, 14, 645. April 5, 1956.

9 FERGUSON J. H. Rupture of the marginal sinus of the placenta. J.A.M.A. Vol. 166, 5: $476,1958$.

10 FERGUSON J. H. HATTON R. L. Abruptio placentae and rupture of the marginal sinus of the placenta: Some Relationships. Am. J. of Obst. \& Gyn. Vol. 78, 5: 947, 1959.

11 FISH J. S., BARTHOLOMEW R. A., COLVIN E. D., GRIMES W. H. The role of marginal sinus rupture in antenatal hemorrhage. Am. J: Obst. \& Gyn. Vol. 61, 1:20. Jan., 1951.

12 GARNEY, H. L., DAVID, G. F. Bleeding in the midtrimestre of pregnancy. Am. J. Obst. \& Gyn. 95: 430, 1966.

13 HARRIS B. A. Jr. Marginal placental bleeding. Am. J. Obst. \& Gyñ. Vol. 64, 1: 61, 1952.

14 LEON JUAN. Tratado de Obstetricia. Vol. 3 Ed. El Ateneo. Buenos Aires. 2a. Ed.

15 NOVAK E., NOVAK E. Gynecologic and Obstetric Pathology. 4th. Edition. W. B. Saunders \& Co. 1958.

16 NOVAK WOODRUFF. Gynecologic and Obstetric Pathology. 5th. Edition. W. B. Saunders \& Co. 1962 .

17 SANCHEZ F. Causas del parto prematuro. Rev. Hospital San Juan de Dios. Bogotá. 7 : 30. 1963.

18 VILLEE, C. A. The placental and fetal membranes. Chap. 3. The Williams and Wilkins Co., 1960. 